\title{
Discrimination between Glioma Grades II and III Using Dynamic Susceptibility Perfusion MRI: A Meta-Analysis
}

(D)Anna F. Delgado and (iD)Alberto F. Delgado

\begin{abstract}
BACKGROUND: DSC perfusion has been evaluated in the discrimination between low-grade and high-grade glioma but the diagnostic potential to discriminate beween glioma grades II and III remains unclear.
\end{abstract}

PURPOSE: Our aim was to evaluate the diagnostic accuracy of relative maximal CBV from DSC perfusion MR imaging to discriminate glioma grades II and III.

DATA SOURCES: A systematic literature search was performed in PubMed/MEDLINE, Embase, Web of Science, and ClinicalTrials.gov.

STUDY SELECTION: Eligible studies reported on patients evaluated with relative maximal CBV derived from DSC with a confirmed neuropathologic diagnosis of glioma World Health Organization grades II and III. Studies reporting on mean or individual patient data were considered for inclusion.

DATA ANALYSIS: Data were analyzed by using inverse variance with the random-effects model and receiver operating characteristic curves describing optimal cutoffs and areas under the curve. Bivariate diagnostic random-effects meta-analysis was used to calculate diagnostic accuracy.

DATA SYNTHESIS: Twenty-eight studies evaluating 727 individuals were included in the meta-analysis. Individual data were available from 10 studies comprising 190 individuals. The mean difference of relative maximal CBV between glioma grades II and III ( $n=727)$ was 1.76 (95\% $\mathrm{CI}, 1.27-2.24 ; P<.001)$. Individual patient data $(n=190)$ had an area under the curve of 0.77 for discriminating glioma grades II and III at an optimal cutoff of 2.02. When we analyzed astrocytomas separately, the area under the curve increased to 0.86 but decreased to 0.61 when we analyzed oligodendrogliomas.

LIMITATIONS: A substantial heterogeneity was found among included studies.

CONCLUSIONS: Glioma grade III had higher relative maximal CBV compared with glioma grade II. A high diagnostic accuracy was found for all patients and astrocytomas; however, the diagnostic accuracy was substantially reduced when discriminating oligodendroglioma grades II and III.

ABBREVIATIONS: QUADAS-2 = Quality Assessment of Diagnostic Accuracy Studies-2; rCBVmax = relative maximum cerebral blood volume; ROC = receiver operating characteristic; WHO $=$ World Health Organization

$\mathbf{P}$ erfusion MR imaging with dynamic susceptibility contrast is a clinical method used to assess brain perfusion in several diseases. ${ }^{1,2}$ DSC has been studied with regard to perfusion in brain tumors such as gliomas, and maximum relative cerebral blood

Received December 7, 2016; accepted after revision March 10, 2017.

From the Department of Clinical Neuroscience (Anna F.D.), Karolinska Institute, Stockholm, Sweden; and Department of Surgical Sciences (Alberto F.D.), Uppsala University, Uppsala, Sweden.

One of the authors (Anna F.D.) received funding from Karolinska University Hospital, Department of Neuroradiology.

Please address correspondence to Anna F. Delgado, MD, PhD, Karolinska Institute, Department of Clinical Neuroscience, Administration, Tomtebodavägen 18A, plan 5, 17177 Stockholm, Sweden. volume (rCBVmax) has been correlated with World Health Organization (WHO) malignant grade ${ }^{3-5}$ to a higher extent than contrast enhancement in morphologic MR imaging. ${ }^{6,7}$ A higher rCBVmax has been associated with high-grade gliomas (WHO grades III and IV) compared with low-grade gliomas (WHO grades I and II)..$^{7-13}$ An optimal cutoff of 1.75 for rCBVmax has been proposed and evaluated in previous studies. ${ }^{714-16}$ However, an optimal cutoff between glioma grades II and III has not been assessed previously, to our knowledge.

The current standard criterion for diagnosing and grading

Indicates article with supplemental on-line tables.

http://dx.doi.org/10.3174/ajnr.A5218 
glioma is through neuropathologic evaluation. ${ }^{17}$ However, not all patients are suitable for surgical resection, and tumor biopsy can lead to a false-negative diagnosis due to an unrepresentative tissue specimen. There is a need for a noninvasive adjunct in discriminating glioma grades at initial diagnosis and at clinical follow-up.

Recently, a meta-analysis studying MR imaging perfusion in low- and high-grade gliomas was published aggregating data on rCBV from DSC imaging, indicating a potential for the clinical use of the method in discrimination of low- and high-grade gliomas. ${ }^{18}$ However, no meta-analysis regarding the specific differences in rCBVmax from DSC imaging between glioma grades II and III has been performed.

Grade II gliomas represent low-grade tumors with longer patient survival and treatment options different from those in highgrade (III) gliomas, which show shorter patient survival and a more dismal prognosis. ${ }^{17,19}$ Furthermore, grade III gliomas have a greater propensity to spread outside their macroscopic margins and are thus harder to resect in total with surgery. ${ }^{20}$ The interest in noninvasive differentiation between glioma grades II and III is attributed to discrepancies in prognosis and treatment options. Noninvasive radiologic evaluation is important in patients not suitable for primary surgery or for longitudinal surveillance in a grade II glioma, with its intrinsic risk of malignant transformation; for treatment follow-up; and as an adjunct to neuropathology.

Several studies analyzing rCBVmax in specific glioma grades have found conflicting results. A number of studies analyzing differences in rCBVmax in glioma grades II and III have failed to show any differences between these malignancy grades. ${ }^{6,21-27} \mathrm{Ra}-$ diologic analyses of differences between low- and high-grade gliomas depend mainly on the specific charactersistic of a glioma grade IV (glioblastoma). The added value of physiologic perfusion MR imaging such as DSC is therefore in the distinction between gliomas of lower grades than WHO grade IV, as in the discrimination between glioma grades II and III. The aim of this meta-analysis was to evaluate differences in relative maximal CBV from DSC perfusion MR imaging between glioma grades II and III and to assess the diagnostic accuracy of rCBVmax to discriminate glioma grades II and III.

\section{MATERIALS AND METHODS}

This meta-analysis was performed according to the Preferred Reporting Items for Systematic Reviews and Meta-Analyses (PRISMA) ${ }^{28}$ statement and current recommended methods for meta-analysis of diagnostic accuracy in imaging journals. $^{29}$

\section{Search Strategy and Information Sources}

Relevant articles were searched from the date of inception to October 2016 in PubMed/MEDLINE, Embase, Web of Science, and ClinicalTrials.gov independently, by the 2 authors (Anna F.D., Alberto F.D.).

PubMed/MEDLINE was searched with the following Medical Subject Heading terms: "glioma perfusion," "MR imaging," "astrocytoma," and "oligodendroglioma." Additional searches were performed with the following terms: "grade," "grading,"
"DSC," "oligoastrocytoma" (the full search strategy is in Online Table 1).

\section{Selection Criteria}

Study selection was based on the patient, intervention, comparison, and outcome criteria, in which eligible studies analyzed patients with glioma WHO grades II and III. The intervention was defined as measurement of rCBVmax from DSC. The comparison was defined as neuropathologic diagnosis after MR imaging of either glioma grade II or III (astrocytoma, oligoastrocytoma, or oligodendroglioma). Studies reporting on mean or individual patient data for grade II and III gliomas were considered for inclusion. Studies reporting on DSC imaging with no restriction in field strength, postprocessing, or ROI method were included. Only English studies were considered. Both retrospective and prospective studies were eligible. Exclusion criteria were the following: studies reporting on patients posttreatment, pediatric patients (younger than 18 years of age), case studies, reviews, abstracts, technical reports, meta-analyses, duplicate or overlapping cohorts, and longitudinal studies. Studies not classifying gliomas according to WHO were excluded.

\section{Data Extraction}

Study characteristics and results were extracted into tables independently by the 2 authors (Anna F.D., Alberto F.D.) with experience in meta-analysis. Data were sought for in the main documents and on-line material.

We extracted the following data: first author, year published, study period, country, perfusion method and MR imaging sequence, MR imaging field strength (1.5T/3T), scanner type (model and company), presaturation of tissue (yes/no), leakagecorrection method (yes/no), WHO classification, study design (prospective/retrospective), number of patients with glioma grades II and III respectively, glioma subtypes (astrocytomas, oligoastrocytomas, oligodendrogliomas), ROI method (rCBVmax or histogram parameter), and mean (SD) rCBVmax in glioma grades II and III, respectively, with associated information about the discriminating potential among grades (yes/no) from the individual study.

Available individual patient data on rCBVmax and neuropathologic diagnosis were extracted separately by the 2 authors, initially blinded to each other's data and subsequently checked for consistency. Patients from overlapping study cohorts (same authors, institution, overlapping years of patient recruitment) were considered for inclusion once in the meta-analysis. Any disagreements regarding data extraction were solved through discussion until consensus.

\section{Data Quality Assessment}

The risk of bias and quality assessment of included studies were independently assessed by the 2 authors (Anna F.D., Alberto F.D.) through the Quality Assessment of Diagnostic Accuracy Studies-2 (QUADAS-2), evaluating patient selection, analysis of index/reference tests, and the flow/timing of the study. ${ }^{30}$ Any disagreements were solved through discussion until consensus. Results from QUADAS-2 were evaluated in sensitivity analysis.

AJNR Am J Neuroradiol 38:1348-55 Jul 2017 www.ajnr.org 


\section{Statistical Analysis}

Mean Study Data. Mean and SDs of rCBVmax from DSC imaging for each glioma grade (WHO grades II or III) were used for the meta-analysis of mean differences. In studies reporting summary estimates other than mean and SD, data were recalculated according to the Cochrane handbook ${ }^{31}$ and Hozo et al. ${ }^{32}$

Syntheses of results were evaluated with a forest plot of mean difference with 95\% CIs of the continuous variable rCBVmax with inverse variance with random effects. The overall effect was calculated with the $z$ score. To assess whether observed differences in results were compatible with chance alone, we calculated the $\chi^{2}$ and its significance level. $\mathrm{I}^{2}$ was calculated to assess any inconsistencies across studies with the estimation of the percentage of the variability in effect estimate due to heterogeneity. The betweenstudy variance in a random-effects meta-analysis was described by $\tau^{2}$.

Additional analyses exploring sources of heterogeneity and the risk of bias were prespecified, except exploratory sensitivity analyses based on QUADAS-2. A funnel plot was calculated for the assessment of publication bias as a scatterplot of included studies with the mean difference in rCBVmax plotted on the horizontal axis, and its standard error, on the vertical axis and visually assessed for asymmetry. ${ }^{33}$

Individual Patient Data. Individual patient data on rCBVmax and neuropathologic diagnosis were analyzed stratified for grade II and III gliomas and in subgroups of histopathologic subtypes (astrocytomas, oligoastrocytomas, and oligodendrogliomas).

To estimate the diagnostic potential of rCBVmax to discriminate glioma grades II and III, we used data from studies including individual patient data to construct receiver operating characteristic (ROC) curves and calculated the area under the curve. From ROC calculations, the number of patients with true-positive, false-negative, false-positive, and true-negative counts was calculated with maximum accuracy set as the cutoff.

The true-positive, false-negative, false-positive, and true-negative counts were analyzed with the bivariate model of mada $^{34}$ in R, Version 3.3.1 (R statistical and computing software; http:// www.r-project.org). ${ }^{35}$ Descriptive forest plots of sensitivity and specificity were constructed. After we assumed a binomial model conditional on the true sensitivity and false-positive rates of the primary studies and a bivariate normal model for the logit-transformed pairs of sensitivities and false-positive rates, a linear mixed model implemented in the Reitsma function of mada was used (bivariate diagnostic random-effects meta-analysis with restricted maximum likelihood estimation method). ${ }^{34}$

Statistical analysis was performed by 1 author (Anna F.D.) with the following statistical programs: RevMan 5.3, ${ }^{36}$ Statistica 12 (https://2ra5-downloads.phpnuke.org/en/c06229/statistica), and R 3.3.1. ${ }^{34,35}$ A continuity correction of 0.5 was used when applicable, and $P<.05$ was considered statistically significant, except in $\chi^{2}$ for which the significance level was set at $P<.10$.

\section{RESULTS}

\section{Literature Search}

The searches yielded 1977 hits (1458 in PubMed, 172 in Embase, 283 in Web of Science, 64 in ClinicalTrials.gov) that were

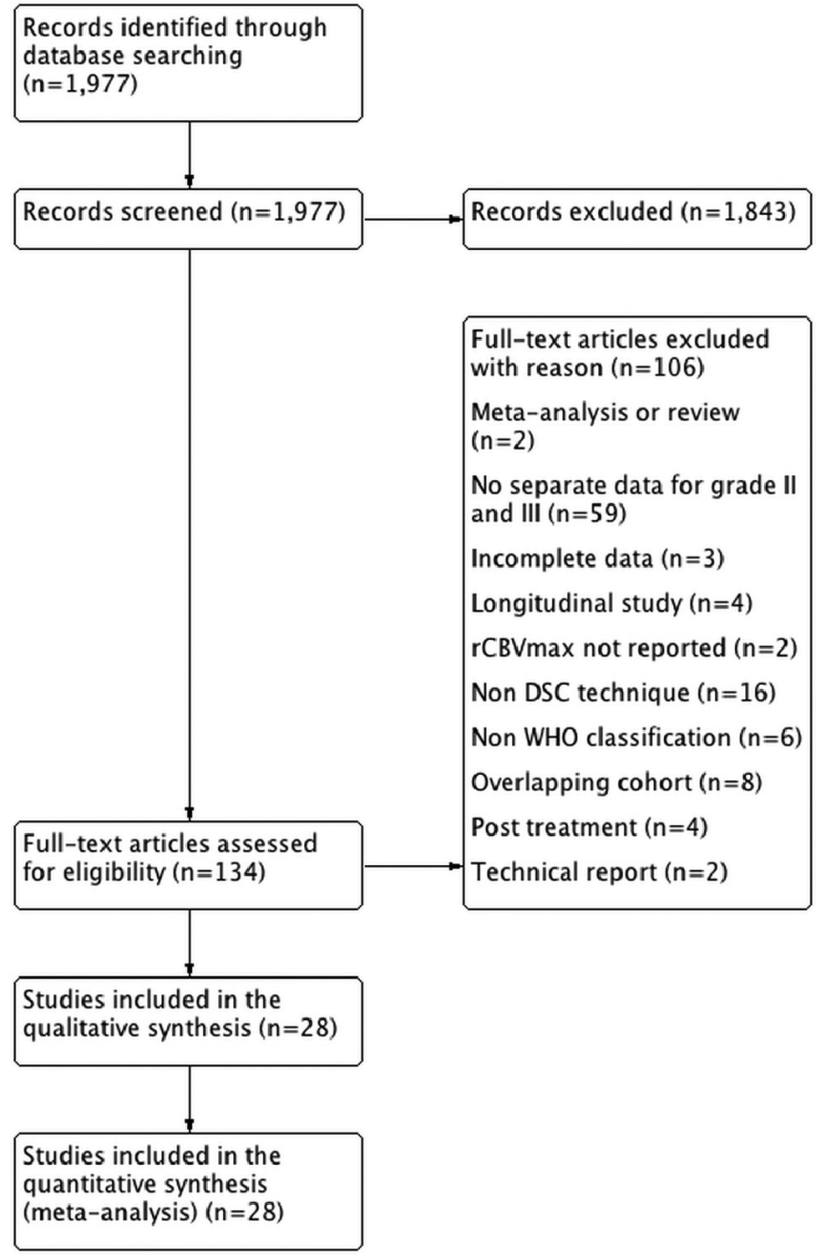

FIG 1. PRISMA flow chart of included and excluded studies.

screened for inclusion in the meta-analysis. Full-text evaluation of 134 articles was performed. Twenty-eight studies comprising 727 patients were included in the qualitative study assessment and quantitative meta-analysis (On-line Table 2). Individual patient data were available in 10 studies comprising 190 patients. A PRISMA flow chart is presented in Fig 1, and the sample search strategy, in On-line Table 1.

\section{Quality Assessment}

Quality assessment from QUADAS-2 is presented in On-line Table 3. Included studies showed a general low risk of bias and a low risk of applicability concerns. Biases included lack of information regarding blinding of index and reference tests and lack of information regarding the time between index and reference tests. Applicability concerns were the use of histogram measures as a surrogate for rCBVmax in 3 studies.

\section{Data Analysis}

Mean Study Data. Tabulated perfusion data and study characteristics of included studies $(n=28)$ evaluating 727 patients are presented in On-line Table 4. The mean (SD) rCBVmax in glioma grade II was 2.14 (1.04); and it was 3.85 (1.18) in grade III (MannWhitney $U$ test, $P<.001)$.

Of the included studies ( $n=28), 16$ showed statistically signifi- 


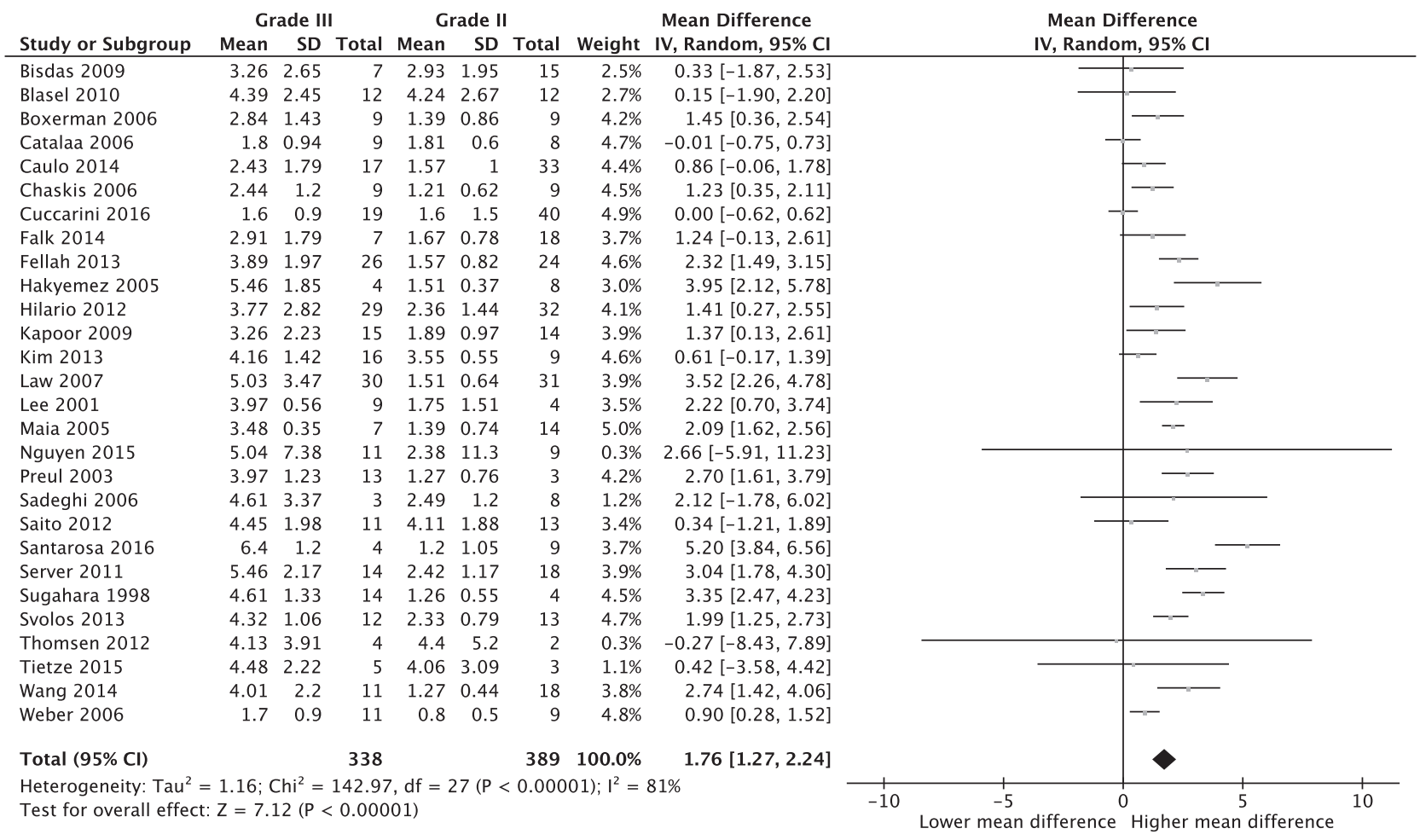

FIG 2. Forest plot of mean differences between glioma grades II and III with a random-effects model.

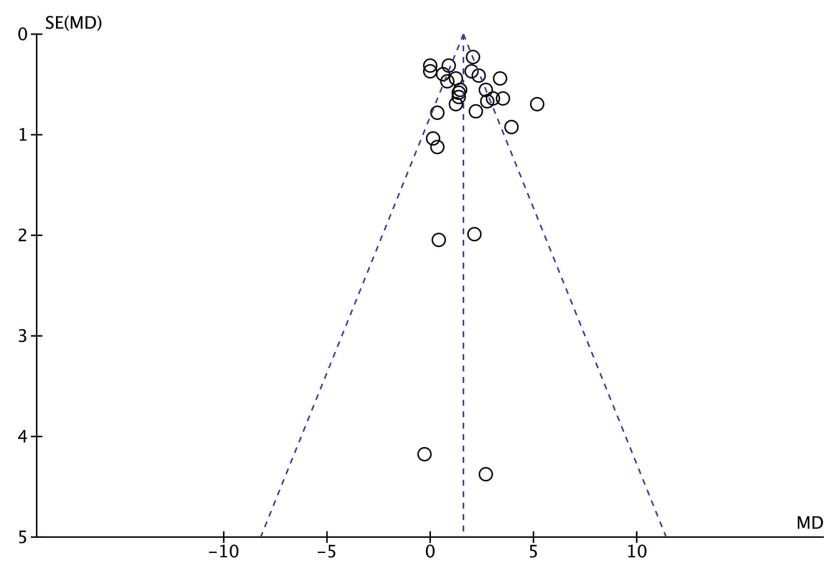

FIG 3. Funnel plot of 28 included studies ( $n=727$ patients) illustrated by open circles with the effect estimate mean difference (MD) of rCBVmax plotted on the horizontal axis, the standard error (SE) of the MD plotted on the vertical axis, and a triangular $95 \%$ confidence region. The study distribution is symmetric without apparent publication bias.

cant differences between glioma grades II and III and 12 studies did not (On-line Table 4). Meta-analytic evaluation of included studies $(n=28)$ showed a mean difference in rCBVmax between glioma grades II and III of 1.76 (95\% CI, 1.27-2.24; $P<.001) ; \mathrm{I}^{2}=81 \%$; Fig 2). Visual assessment of the funnel plot revealed no evidence of the existence of publication bias (Fig 3).

Heterogeneity between study results $\left(\chi^{2}\right)$, inconsistencies between studies $\left(\mathrm{I}^{2}\right)$, and between-study variances $\left(\tau^{2}\right)$ are presented in the Table. Three study characteristics increasing heterogeneity $\left(\chi^{2}\right)$ across studies were significantly related to studies without published individual patient data $\left(n=121, \chi^{2}=18, P=.07\right)$ and ROI analysis $\left(n=25, \chi^{2}=116, P<.001\right)$ and studies with a blinded index test evaluation ( $\left.n=14, \chi^{2}=98, P<.001\right)$. Study characteristics increasing inconsistencies across studies $\left(\mathrm{I}^{2}\right)$ were the following: prospective study design $\left(n=11, \mathrm{I}^{2}=89\right)$, optimal flow and timing assessed in QUADAS-2 $\left(n=7, \mathrm{I}^{2}=\right.$ $88)$, and blinded index test evaluation $\left(n=14, \mathrm{I}^{2}=87\right)$. Study characteristics with increasing $\chi^{2}$ were those with correction for contrast agent leakage according to Boxerman et $\mathrm{al}^{1}(n=8$, $\left.\tau^{2}=2.58\right)$, those reporting on WHO 2007 classification $(n=$ $\left.11, \tau^{2}=2.07\right)$, and those with a prospective study design ( $n=$ $\left.11, \tau^{2}=1.94\right)$.

Individual Patient Data. A total of 190 patients (105 with WHO grade II, 85 with WHO grade III) from 10 studies reported on individual patient data (On-line Table 5). ROC analysis showed discriminatory potential between glioma grades II and III ( $n=$ 190 ) with an area under curve of 0.77 with maximum accuracy at an exploratory cutoff of 2.02 (Fig 4). Data were stratified for tumor type: astrocytomas $(n=102)$, with an area under curve of 0.86 and maximum accuracy at cutoff at 2.02, oligoastrocytomas $(n=28)$ with an area under curve of 0.83 and maximum accuracy at cutoff at 3.19 , and oligodendrogliomas $(n=60)$ with an area under curve of 0.61 and maximum accuracy at cutoff at 6.23 .

Data on true-positive, false-negative, false-positive, and truenegative counts from each study are presented in On-line Table 6. Univariate paired forest plots for individual patient data are presented in Fig $5 A,-B$.

A bivariate diagnostic random-effects meta-analysis model with a restricted maximum likelihood estimation method described the summary receiver operating characteristic curve in Fig 6 with a sensitivity of 0.71 (95\% CI, $0.48-0.86)$ and a false-positive rate of 0.15 (95\% CI, 0.09-0.25). 


\begin{tabular}{|c|c|c|c|c|}
\hline Factor (No. of Studies) & $\tau^{2}$ & $\chi^{2}(P)$ & $\mathrm{I}^{2}(\%)$ & $\begin{array}{l}\text { Mean Differences } \\
(95 \% \mathrm{Cl}) \text { Random }\end{array}$ \\
\hline Studies without significant differences between grades II and III $(n=14)$ & 0.00 & $6.5(.84)$ & 0 & $0.35(0.01-0.69)$ \\
\hline Oligodendroglial tumors $(n=3)$ & 0.04 & $2.17(.34)$ & 8 & $1.95(1.21-2.96)$ \\
\hline Histogram rCBVmax $(n=3)$ & 0.10 & $2.88(.24)$ & 31 & $0.45(-0.17-1.08)$ \\
\hline Published individual patient data $(n=10)$ & 0.28 & $15.76(<.001)$ & 43 & $1.82(1.28-2.36)$ \\
\hline Retrospective study design $(n=17)$ & 0.70 & $52.34(<.001)$ & 69 & $1.42(0.89-1.96)$ \\
\hline Astrocytic tumors $(n=6)$ & 1.57 & $20.69(<.001)$ & 76 & $2.28(0.96-3.60)$ \\
\hline Studies with significant differences between grades II and III ( $n=16)$ & 0.73 & $63.01(<.001)$ & 76 & $2.37(1.87-2.87)$ \\
\hline Non-WHO 2007 classification ( $n=17)$ & 0.88 & $73.74(<.001)$ & 78 & $1.71(1.16-2.26)$ \\
\hline No correction for contrast agent leakage according to Boxerman $(n=20)$ & 0.88 & $86.07(<.001)$ & 78 & $1.75(1.24-2.25)$ \\
\hline ROI analysis $(n=25)$ & 1.09 & $116.10(<.001)$ & 79 & $1.93(1.43-2.44)$ \\
\hline Studies published in 2010 or before $(n=14)$ & 1.02 & $65.67(<.001)$ & 80 & $1.81(1.17-2.44)$ \\
\hline No presaturation of tissue $(n=18)$ & 1.05 & $84.70(<.001)$ & 80 & $1.85(1.27-2.43)$ \\
\hline All studies $(n=28)$ & 1.16 & $142.97(<.001)$ & 81 & $1.76(1.27-2.24)$ \\
\hline $3 \mathrm{~T}(n=10)$ & 1.67 & $46.71(<.001)$ & 81 & $1.66(0.69-2.64)$ \\
\hline Presaturation of tissue $(n=10)$ & 1.53 & $53.21(<.001)$ & 83 & $1.60(0.68-2.15)$ \\
\hline Studies published after $2010(n=14)$ & 1.50 & $75.04(<.001)$ & 83 & $1.70(0.93-2.48)$ \\
\hline $1.5 T(n=16)$ & 1.17 & $95.95(<.001)$ & 84 & $1.83(1.22-2.45)$ \\
\hline WHO 2007 classification $(n=11)$ & 2.07 & $68.39(<.001)$ & 85 & $1.85(0.85-2.86)$ \\
\hline No published individual patient data $(n=18)$ & 1.54 & $120.79(.07)$ & 86 & $1.75(1.07-2.42)$ \\
\hline Blinded reference test evaluation $(n=5)$ QUADAS-2 & 1.10 & $29.23(<.001)$ & 86 & $1.30(0.29-2.32)$ \\
\hline Correction for leakage according to Boxerman $(n=8)$ & 2.58 & $54.12(<.001)$ & 87 & $1.83(0.49-3.16)$ \\
\hline Blinded index test evaluation $(n=14)$ QUADAS-2 & 1.57 & $97.79(<.001)$ & 87 & $1.77(1.03-2.51)$ \\
\hline Optimal flow and timing $(n=7)$ QUADAS-2 & 1.57 & $49.31(<.001)$ & 88 & $1.69(0.57-2.80)$ \\
\hline Prospective study design $(n=11)$ & 1.94 & $89.9(<.001)$ & 89 & $2.27(1.35-3.20)$ \\
\hline
\end{tabular}

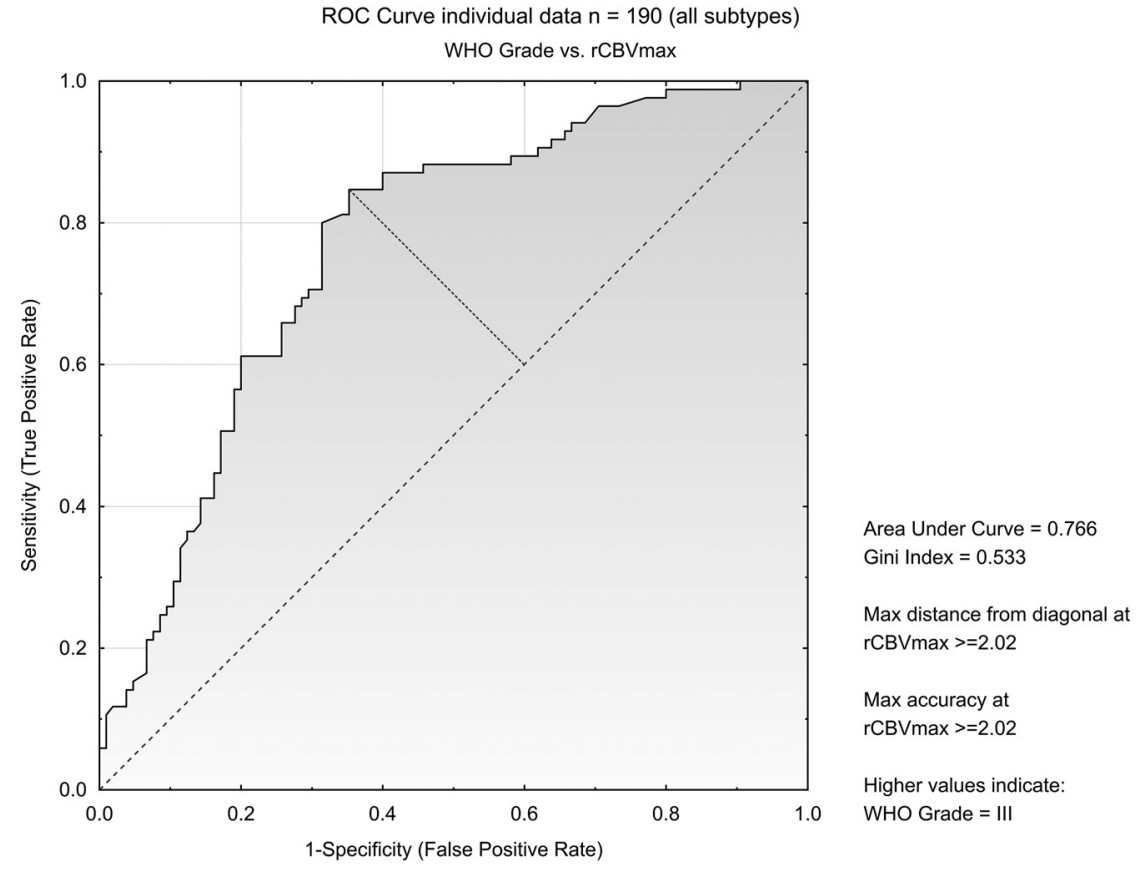

FIG 4. ROC curve on individual patient data from 10 studies including all patients $(n=190)$.

\section{DISCUSSION}

This meta-analysis investigated the potential of DSC perfusion MR imaging to distinguish glioma grades II and III. Our study showed significant differences in rCBVmax between glioma grades II and III, with higher rCBVmax in WHO grade III compared with grade II. Furthermore, we evaluated individual patient data on rCBVmax to explore the diagnostic potential and the optimal cutoff for rCBVmax between glioma grades II and III, stratified for glial subtypes.
The importance of this meta-analysis is mainly that it describes the differences in rCBVmax between glioma grades II and III and that these differences can be measured and applied in a clinical setting. This evidence is based on data from 28 studies including 727 individual patients. This is an interesting finding in a field with small conflicting studies. Noninvasive glioma grade evaluation is an important adjunct to neuropathologic evaluation in patients deemed inoperable or at longitudinal follow-up. It might also be of value in patients with nonrepresentative biopsies that might lead to a false-negative diagnosis. Furthermore, the pooling of data across studies adds precision to estimates and can guide future work.

The quality and bias assessment of included studies showed intermediateto-low risk of bias and an intermediateto-high study quality. However, minor concerns about blinding of index and reference tests, flow and timing in the studies, and the lack of prespecified cutoffs in ROC analysis could have affected the results of the meta-analysis. Although no asymmetry was detected in the funnel plot, evidence of greay literature was searched in ClinicalTrials.gov.

Statistical heterogeneity between studies was substantial. Potential sources for heterogeneity were evaluated but did not alter the main study finding of statistical differences in $\mathrm{rCBV}$ max between glioma grades II and III, except for sensitivity analyses of 3 studies 


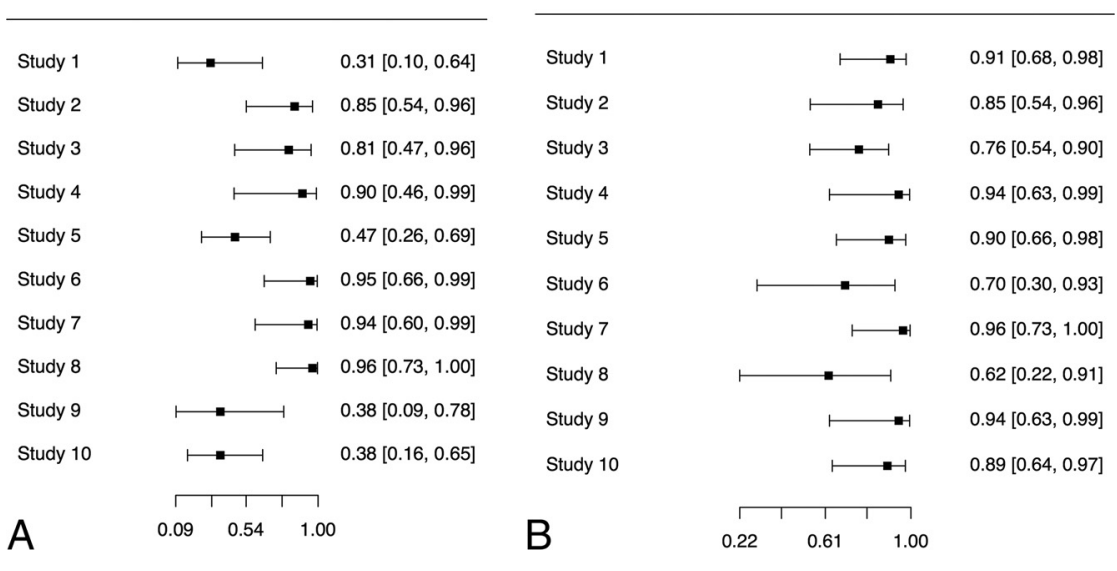

FIG 5. Paired forest plots for individual patient data $(n=190)$. A, Forest plot of sensitivity. $B$, Forest plot of specificity.

\section{SROC curve (bivariate model) for data}

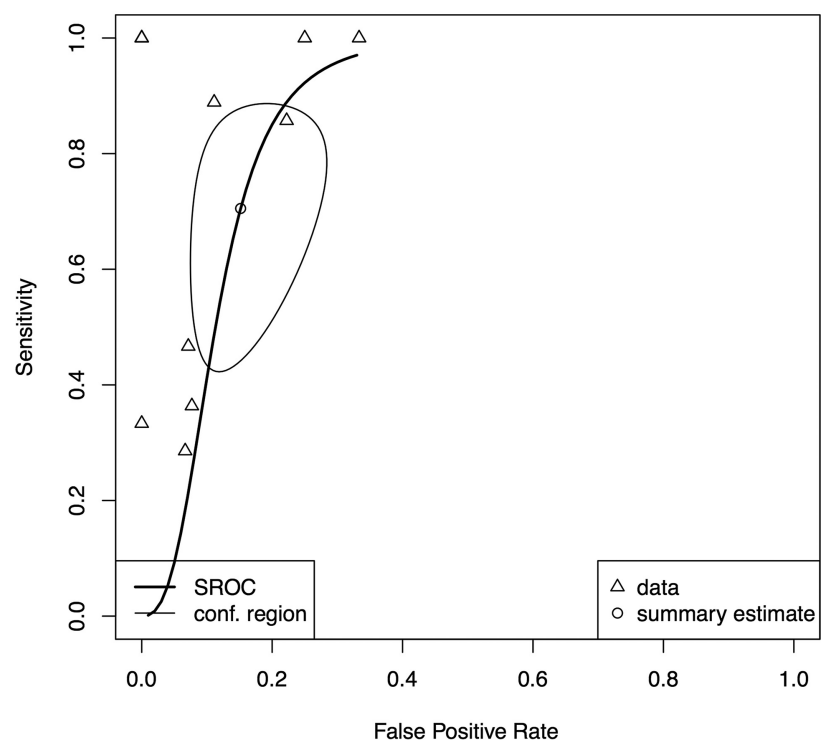

FIG 6. Graph showing a point estimate of the pair of sensitivity and false-positive rates plotted together with a confidence region, without extrapolation beyond the range of the original data (individual patient data, $n=190)$.

with a histogram technique to identify rCBVmax, which showed no statistically significant difference in rCBVmax between groups of glioma grades II and III. ${ }^{10,21,37}$

Analyzing specific subtypes of glioma grades II and III preoperatively is counterintuitive because subtype attribution is largely unknown before neuropathologic evaluation. Knowledge of differences in rCBVmax between subtypes of gliomas relates to discrepancies in biology, more than it adds to the diagnostic potential in a preoperative clinical setting. ${ }^{38-41}$ However, while sensitivity analysis of studies analyzing oligodendroglial tumors (oligoastrocytomas or oligodendrogliomas) showed differences in rCBVmax among tumor grades similar to those in astrocytomas, the individual patient data analysis stratifying oligodendroglial tumors into oligoastrocytomas and oligodendrogliomas decreased the diagnostic potential when analyzing oligodendrogliomas separately.

DSC might be of added value at the longitudinal follow-up in specific glioma subtypes after initial neuropathologic diagnosis when assessing malignant transformation. Preoperatively when the tumor subtype is unknown (or only suspected based on the morphologic appearance on CT or MR imaging), a true diagnostic discrepancy by rCBVmax between glioma grades II and III is possibly hampered by the lack of information regarding glial cell subtype. Speaking for the applicability of DSC is the, relative to astrocytomas, lower incidence of oligodendrogliomas, making this method important in most patients.

When evaluating the diagnostic accuracy of DSC in this meta-analysis, we used exploratory evaluations of optimal cutoffs for discrimination between glioma grades II and III. This lack of evaluating diagnostic accuracy from a prespecified cutoff can increase the diagnostic potential presented in the meta-analysis. A limitation in DSC perfusion MR imaging is the nonlinear relationship between gadolinium concentration and the relaxivity-time curve, and nonexponential relaxation decay. ${ }^{42}$ Consequently, measured rCBV values, despite normalization to healthy white matter, are dependent on TE, injection rate, and total dose and gadolinium preloading and act as potential drivers for heterogeneity. ${ }^{43}$

When discussing an optimal cutoff between glioma grades II and III, we found maximum accuracy from ROC analysis at rCB$\operatorname{Vmax}=2.02$ (individual patient data, No. of patients $=190$, No. of studies $=10)$. This is a higher cutoff than previously suggested for optimal discrimination between low- (WHO I and II) and high-grade gliomas (WHO III and IV), which might be explained by different compositions of specific grades and subtypes in the groups of low- and high-grade gliomas in previously published material. This cutoff needs to be validated in prospective studies with standardized methodology, taking the differences in biology between glioma subtypes into account.

On the basis of subgroup analyses, the new molecular advances and WHO 2016 classification are applicable to our results regarding astrocytomas and oligodendrogliomas. The main difference pertains to molecular classification of oligoastrocytomas as either astrocytomas or oligodendrogliomas, which are analyzed separately in the individual patient data analysis. ${ }^{17}$

\section{CONCLUSIONS}

This meta-analysis evaluates DSC MR imaging perfusion in patients with glioma grades II and III. A higher rCBVmax was found in glioma grade III compared with grade II $(n=727)$. A high diagnostic accuracy was found for all patients and when analyzing astrocytomas separately; however, the diagnostic accuracy was substantially reduced when discriminating grade II and III oligodendrogliomas.

\section{REFERENCES}

1. Boxerman JL, Schmainda KM, Weisskoff RM. Relative cerebral blood volume maps corrected for contrast agent extravasation sig-

AJNR Am J Neuroradiol 38:1348-55 Jul 2017 www.ajnr.org

1353 
nificantly correlate with glioma tumor grade, whereas uncorrected maps do not. AJNR Am J Neuroradiol 2006;27:859-67 Medline

2. Boxerman JL, Shiroishi MS, Ellingson BM, et al. Dynamic susceptibility contrast MR imaging in glioma: review of current clinical practice. Magn Reson Imaging Clin N Am 2016;24:649-70 CrossRef Medline

3. Law M, Young R, Babb J, et al. Histogram analysis versus region of interest analysis of dynamic susceptibility contrast perfusion MR imaging data in the grading of cerebral gliomas. AJNR Am J Neuroradiol 2007;28:761-66 Medline

4. Hilario A, Ramos A, Perez-Nuñez A, et al. The added value of apparent diffusion coefficient to cerebral blood volume in the preoperative grading of diffuse gliomas. AJNR Am J Neuroradiol 2012;33: 701-07 CrossRef Medline

5. Wang XC, Zhang H, Tan Y, et al. Combined value of susceptibilityweighted and perfusion-weighted imaging in assessing WHO grade for brain astrocytomas. J Magn Reson Imaging 2014;39:1569-74 CrossRef Medline

6. Caulo M, Panara V, Tortora D, et al. Data-driven grading of brain gliomas: a multiparametric MR imaging study. Radiology 2014;272: 494-503 CrossRef Medline

7. Law M, Yang S, Wang H, et al. Glioma grading: sensitivity, specificity, and predictive values of perfusion MR imaging and proton MR spectroscopic imaging compared with conventional MR imaging. AJNR Am J Neuroradiol 2003;24:1989-98 Medline

8. Park MJ, Kim HS, Jahng GH, et al. Semiquantitative assessment of intratumoral susceptibility signals using non-contrast-enhanced high-field high-resolution susceptibility-weighted imaging in patients with gliomas: comparison with MR perfusion imaging. AJNR Am J Neuroradiol 2009;30:1402-08 CrossRef Medline

9. Van Cauter S, De Keyzer F, Sima DM, et al. Integrating diffusion kurtosis imaging, dynamic susceptibility-weighted contrast-enhanced MRI, and short echo time chemical shift imaging for grading gliomas. Neuro Oncol 2014;16:1010-21 CrossRef Medline

10. Kim H, Choi SH, Kim JH, et al. Gliomas: application of cumulative histogram analysis of normalized cerebral blood volume on $3 \mathrm{~T}$ MRI to tumor grading. PLoS One 2013;8:e63462 CrossRef Medline

11. Emblem KE, Scheie D, Due-Tonnessen P, et al. Histogram analysis of MR imaging-derived cerebral blood volume maps: combined glioma grading and identification of low-grade oligodendroglial subtypes. AJNR Am J Neuroradiol 2008;29:1664-70 CrossRef Medline

12. Di Costanzo A, Pollice S, Trojsi F, et al. Role of perfusion-weighted imaging at 3 Tesla in the assessment of malignancy of cerebral gliomas [In English, Italian]. Radiol Med 2008;113:134-43 CrossRef Medline

13. Law M, Young R, Babb J, et al. Comparing perfusion metrics obtained from a single compartment versus pharmacokinetic modeling methods using dynamic susceptibility contrast-enhanced perfusion MR imaging with glioma grade. AJNR Am J Neuroradiol 2006; 27:1975-82 Medline

14. Caseiras GB, Chheang S, Babb J, et al. Relative cerebral blood volume measurements of low-grade gliomas predict patient outcome in a multi-institution setting. Eur J Radiol 2010;73:215-20 CrossRef Medline

15. Nasseri M, Gahramanov S, Netto JP, et al. Evaluation of pseudoprogression in patients with glioblastoma multiforme using dynamic magnetic resonance imaging with ferumoxytol calls RANO criteria into question. Neuro Oncol 2014;16:1146-54 CrossRef Medline

16. Hu LS, Kelm Z, Korfiatis P, et al. Impact of software modeling on the accuracy of perfusion MRI in glioma. AJNR Am J Neuroradiol 2015; 36:2242-49 CrossRef Medline

17. Louis DN, Perry A, Reifenberger G, et al. The $\mathbf{2 0 1 6}$ World Health Organization Classification of Tumors of the Central Nervous System: a summary. Acta Neuropathol 2016;131:803-20 CrossRef Medline

18. Usinskiene J, Ulyte A, Bjornerud A, et al. Optimal differentiation of high- and low-grade glioma and metastasis: a meta-analysis of per- fusion, diffusion, and spectroscopy metrics. Neuroradiology 2016; 58:339-50 CrossRef Medline

19. Crocetti E, Trama A, Stiller C, et al. Epidemiology of glial and non-glial brain tumours in Europe. Eur J Cancer 2012;48:1532-42 CrossRef Medline

20. Kelly PJ, Daumas-Duport C, Kispert DB, et al. Imaging-based stereotaxic serial biopsies in untreated intracranial glial neoplasms. J Neurosurg 1987;66:865-74 CrossRef Medline

21. Catalaa I, Henry R, Dillon WP, et al. Perfusion, diffusion and spectroscopy values in newly diagnosed cerebral gliomas. NMR Biomed 2006;19:463-75 CrossRef Medline

22. Cuccarini V, Erbetta A, Farinotti M, et al. Advanced MRI may complement histological diagnosis of lower grade gliomas and help in predicting survival. J Neurooncol 2016;126:279-88 CrossRef Medline

23. Nguyen TB, Cron GO, Perdrizet K, et al. Comparison of the diagnostic accuracy of DSC- and dynamic contrast-enhanced MRI in the preoperative grading of astrocytomas. AJNR Am J Neuroradiol 2015; 36:2017-22 CrossRef Medline

24. Saito T, Yamasaki F, Kajiwara Y, et al. Role of perfusion-weighted imaging at $3 \mathrm{~T}$ in the histopathological differentiation between astrocytic and oligodendroglial tumors. Eur J Radiol 2012;81:1863-69 CrossRef Medline

25. Thomsen H, Steffensen E, Larsson EM. Perfusion MRI (dynamic susceptibility contrast imaging) with different measurement approaches for the evaluation of blood flow and blood volume in human gliomas. Acta Radiol 2012;53:95-101 CrossRef Medline

26. Toyooka M, Kimura H, Uematsu H, et al. Tissue characterization of glioma by proton magnetic resonance spectroscopy and perfusionweighted magnetic resonance imaging: glioma grading and histological correlation. Clin Imaging 2008;32:251-58 CrossRef Medline

27. Togao O, Hiwatashi A, Yamashita K, et al. Grading diffuse gliomas without intense contrast enhancement by amide proton transfer MR imaging: comparisons with diffusion- and perfusion-weighted imaging. Eur Radiol 2017;27:578-88 CrossRef Medline

28. Moher D, Liberati A, Tetzlaff J, et al. Preferred reporting items for systematic reviews and meta-analyses: the PRISMA statement. $B M$ J 2009;339:b2535 CrossRef Medline

29. McGrath TA, McInnes MD, Korevaar DA, et al. Meta-analyses of diagnostic accuracy in imaging journals: analysis of pooling techniques and their effect on summary estimates of diagnostic accuracy. Radiology 2016;281:78-85 CrossRef Medline

30. Whiting PF, Rutjes AW, Westwood ME, et al. QUADAS-2: a revised tool for the quality assessment of diagnostic accuracy studies. Ann Intern Med 2011;155:529-36 CrossRef Medline

31. Higgins JP, Green S. Cochrane Handbook for Systematic Reviews of Interventions, Version 5.1.0 (updated March 2011). The Cochrane Collaboration, 2011. http://handbook.cochrane.org/. Accessed September 6, 2016

32. Hozo SP, Djulbegovic B, Hozo I. Estimating the mean and variance from the median, range, and the size of a sample. $B M C$ Med Res Methodol 2005;5:13 CrossRef Medline

33. Light RJ, Pillemer DB. Summing Up: The Science of Reviewing Research. Cambridge: Harvard University Press; 1984

34. Doebler P, Holling H. Meta-Analysis of Diagnostic Accuracy with mada. 2015. https://cran.r-project.org/web/packages/mada/vignettes/ mada.pdf. Accessed September 6, 2016

35. R Development Core Team. R: A Language and Environment for Statistical Computing. Vienna: R Foundation for Statistical Computing; 2010

36. Review Manager (RevMan). 5.3 ed. Copenhagen: The Nordic Cochrane Centre: The Cochrane Collaboration; 2014 http://www. medsci.cn/webeditor/uploadfile/201408/20140815214316360.pdf. Accessed September 6, 2016

37. Falk A, Fahlström M, Rostrup E, et al. Discrimination between glioma grades II and III in suspected low-grade gliomas using dynamic contrast-enhanced and dynamic susceptibility contrast perfusion MR imaging: a histogram analysis approach. Neuroradiology 2014;56:1031-38 CrossRef Medline

38. Cairncross JG, Wang M, Jenkins RB, et al. Benefit from procarba- 
zine, lomustine, and vincristine in oligodendroglial tumors is associated with mutation of IDH. J Clin Oncol 2014;32:783-90 CrossRef Medline

39. van den Bent MJ, Brandes AA, Taphoorn MJ, et al. Adjuvant procarbazine, lomustine, and vincristine chemotherapy in newly diagnosed anaplastic oligodendroglioma: long-term follow-up of EORTC brain tumor group study 26951. J Clin Oncol 2013;31:344-50 CrossRef Medline

40. Eckel-Passow JE, Lachance DH, Molinaro AM, et al. Glioma groups based on $1 \mathrm{p} / 19 \mathrm{q}, \mathrm{IDH}$, and TERT promoter mutations in tumors. N Engl J Med 2015;372:2499-508 CrossRef Medline
41. Brat DJ, Verhaak RG, Aldape KD et al; Cancer Genome Atlas Research Network. Comprehensive, integrative genomic analysis of diffuse lower-grade gliomas. N Engl J Med 2015;372:2481-98 CrossRef Medline

42. Kiselev VG, Posse S. Analytical model of susceptibility-induced MR signal dephasing: effect of diffusion in a microvascular network. Magn Reson Med 1999;41:499-509 Medline

43. Yablonskiy DA, Haacke EM. Theory of NMR signal behavior in magnetically inhomogeneous tissues: the static dephasing regime. Magn Reson Med 1994;32:749-63 CrossRef Medline 